\section{A) Check for updates}

Cite this: Analyst, 2021, 146, 4605

\title{
Development and validation of a point-of-care breath test for octane detection $\uparrow$
}

\author{
Laura A. Hagens, (D)*a Alwin R. M. Verschueren, ${ }^{b}$ Ariana Lammers, (D) ${ }^{c}$

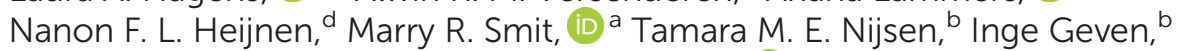 \\ Marcus J. Schultz, ${ }^{a, e, f}$ Dennis C. J. J. Bergmans, (D) ${ }^{d, g}$ Ronny M. Schnabel ${ }^{d}$ and \\ Lieuwe D. J. Bos ${ }^{\star a, c}$
}

\begin{abstract}
Background: There is a demand for a non-invasive bedside method to diagnose Acute Respiratory Distress Syndrome (ARDS). Octane was discovered and validated as the most important breath biomarker for diagnosis of ARDS using gas-chromatography and mass-spectrometry (GC-MS). However, GC-MS is unsuitable as a point-of-care (POC) test in the intensive care unit (ICU). Therefore, we determined if a newly developed POC breath test can reliably detect octane in exhaled breath of invasively ventilated ICU patients. Methods: Two developmental steps were taken to design a POC breath test that relies on gas-chromatography using air as carrier gas with a photoionization detector. Calibration measurements were performed with a laboratory prototype in healthy subjects. Subsequently, invasively ventilated patients were included for validation and assessment of repeatability. After evolving to a POC breath test, this device was validated in a second group of invasively ventilated patients. Octane concentration was based on the area under the curve, which was extracted from the chromatogram and compared to known values from calibration measurements. Results: Five healthy subjects and 53 invasively ventilated patients were included. Calibration showed a linear relation $\left(R^{2}=1.0\right)$ between the octane concentration and the quantified octane peak in the low parts per billion (ppb) range. For the POC breath test the repeatability was excellent $\left(R^{2}=0.98, I C C=0.97(95 \% \mathrm{Cl}\right.$ 0.94-0.99)). Conclusion: This is the first study to show that a POC breath test can rapidly and reliably detect octane, with excellent repeatability, at clinically relevant levels of low ppb in exhaled breath of ventilated ICU patients. This opens possibilities for targeted exhaled breath analysis to be used as a bedside test and makes it a potential diagnostic tool for the early detection of ARDS.
\end{abstract}

Received 3rd March 2021 Accepted 10th June 2021 DOI: $10.1039 / \mathrm{d} 1 \mathrm{an} 00378$ j rsc.li/analyst

\section{Introduction}

Acute respiratory distress syndrome (ARDS) is a severe complication of critical illness, that is characterized by acute diffuse lung injury resulting in increased pulmonary vascular permeability. ${ }^{1}$ ARDS is associated with hospital mortality rates of around $40 \%{ }^{2}$ Diagnosis of ARDS according to the Berlin definition is based on radiological, clinical and physiological criteria. ${ }^{1}$ The criteria need further improvement because they are aspecific, subjective and reflect a late stage of the disease. ${ }^{3}$ For example, interpretation of chest radiography is known to have a poor inter-observer reliability. ${ }^{4}$ However, a bedside test which is preferably objective, non-invasive, and rapid has not been developed yet. ${ }^{5}$

Using gas chromatography and mass spectrometry (GC-MS) for unbiased metabolomics analysis, octane was discovered and validated as the most important breath biomarker for the diagnosis of ARDS. ${ }^{5,6}$ GC-MS has limited potential to be used as a point-of-care (POC) test in the intensive care unit (ICU) as the machine is complex to handle and expensive to operate. ${ }^{7}$ Important limiting aspects are the need to maintain a vacuum, 
a steady supply of helium, the variation in detector sensitivity and challenges in the interpretation of data output.

We developed and tested a POC test for $n$-octane (from here onwards referred to as octane) detection in breath of invasively ventilated ICU patients. This device should be able to detect octane with high sensitivity, in ranges of low parts per billion ( $\mathrm{ppb}$ ), without the need for a helium source or a vacuum. We investigated the repeatability and robustness of the POC breath test in detecting octane, first in exhaled breath of healthy controls and subsequently in breath of invasively ventilated ICU patients. We hypothesized that it is possible to reliably detect octane in exhaled breath of invasively ventilated ICU patients using the POC breath test.

\section{Methods}

\section{Study design}

Table 1 outlines the steps taken in this study to get from a bench model to a bedside POC breath test. In the bench study, a laboratory prototype was calibrated and validated using calibration samples with known concentrations of octane. Subsequently, measurements in healthy subjects were used to validate the performance of the prototype. Thereafter, breath from invasively ventilated patients was collected in an observational cohort study. The samples were transported to the laboratory for measurements with the prototype to assess repeatability, and for measurements with gold standard GC-MS to assess the reproducibility (Table 1 ).

After the bench study, prior to the subsequent bedside study, a period was taken to further develop this prototype into a POC prototype, described as the POC breath test, that could be used in the ICU. Validation and calibration was performed with calibration samples using a mixture of known components with a prespecified concentration.

During the bedside study, breath from invasively ventilated patients was collected as part of an observational cohort study. These samples were analysed on-site, in the ICU, using the POC breath test in order to validate the repeatability and implement the technique into the clinical setting (Table 1). Again GC-MS was used, to analyse the reproducibility.

\section{Subjects and ethical considerations}

For the calibration of the laboratory prototype 5 healthy volunteers were included. The Internal Committee on Biomedical Experiments (ICBE) of Philips Research, Eindhoven, The Netherlands, approved this part of the study. Informed consent was obtained for exhaled breath collection from the healthy volunteers.

For clinical implementation and validation, consecutive patients admitted to the ICU of Amsterdam UMC, location AMC, were screened for eligibility in two periods. To validate the laboratory prototype, the inclusion period ranged from April to July 2017. To validate the POC breath test, the second round of inclusions was from February to March 2020. Newly admitted intubated and invasively ventilated patients who met the following criteria were included: expected duration of invasive ventilation of at least 24 hours and age above 18 years. Exclusion criteria were previous invasive ventilation ( $>48$ hours during last 7 days) and tracheostomy. Additionally, patients with highly contagious pathogens necessitating strict isolation

Table 1 Study design with steps taken to develop bench model into bedside POC breath test

\begin{tabular}{|c|c|c|c|c|c|c|}
\hline Translational step & \multicolumn{3}{|c|}{ Bench } & \multirow{4}{*}{$\begin{array}{l}\text { T) } \\
\frac{\delta}{\leq} \\
\overline{0} \\
\overline{\frac{\partial}{g}}\end{array}$} & \multirow{2}{*}{\multicolumn{2}{|c|}{$\begin{array}{c}\text { Bedside } \\
2\end{array}$}} \\
\hline Development step & & \multicolumn{2}{|c|}{1} & & & \\
\hline Period & & \multicolumn{2}{|c|}{ April - July 2017} & & \multicolumn{2}{|c|}{ February - March 2020} \\
\hline Research aim & Calibration & \multicolumn{2}{|c|}{ Repeatability } & & \multicolumn{2}{|c|}{ Repeatability and implementation } \\
\hline Device & $\begin{array}{l}\text { Laboratory } \\
\text { prototype }\end{array}$ & $\begin{array}{l}\text { Laboratory } \\
\text { prototype }\end{array}$ & GC-MS & \multirow{5}{*}{ 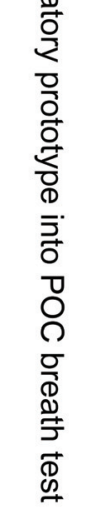 } & POC breath test & GC-MS \\
\hline Subjects & $\begin{array}{l}5 \text { Healthy } \\
\text { subjects }\end{array}$ & \multicolumn{2}{|c|}{40 Patients } & & \multicolumn{2}{|c|}{13 Patients } \\
\hline Location analysis & Laboratory & \multicolumn{2}{|c|}{ Laboratory } & & \multicolumn{2}{|c|}{ On-site } \\
\hline $\begin{array}{c}\text { Number of } \\
\text { measurements }\end{array}$ & 30 & 40 duplicates & 40 duplicates & & 30 duplicates & 30 duplicates \\
\hline Type of tube used & \multicolumn{3}{|c|}{ Tenax GR and Carbograph 5TD } & & \multicolumn{2}{|c|}{ Tenax GR and Carbograph 5TD } \\
\hline Octane source & External & \multicolumn{2}{|c|}{ Breath of patient } & & \multicolumn{2}{|c|}{ Breath of patient } \\
\hline
\end{tabular}


were excluded. The Institutional Review Board of the Academic Medical Center (Amsterdam, the Netherlands), reviewed the study protocol and judged that the study procedures were fully non-invasive and did not require informed consent according to the Medical Research Involving Human Subjects Act (W18_311 \# 18.358). In line with the European Union General Data Protection Regulation, written informed consent was obtained for data usage.

\section{Study procedure}

Calibration. The laboratory prototype was calibrated with variable amounts of octane in calibration samples and in breath samples of healthy subjects. Based on the discovery and validation study in invasively ventilated patients with and without ARDS the concentration was expected to be in the 0.1-0.4 ppb range for patients without ARDS and between 0.2 and 2 ppb for patients with ARDS. ${ }^{6}$ To ascertain that higher values were also covered we also analysed concentrations up to $8 \mathrm{ppb}$ in the laboratory prototype. The calibration samples for the POC breath test were loaded onto the same type of sorbent tubes as used for the breath samples. After thermal annealing, the sorbent tubes were loaded with a fixed volume of $0.4 \mathrm{~mL}$ of $1 \mathrm{ppm}$ TO-15 mixture (25 component subset, Scotty, United States) plus a variable amount (between 1-5 ng) of $n$-octane from a certified permeation tube (Kintek, United States). Leading to calibration samples with concentrations of 0.2, 0.5, 1.0 and 2.0 ppb.

Breath sampling in healthy subjects. Sampling of exhaled breath in healthy subjects was performed using 3L Teflon bags (Scentroid, Canada). First these were filled with synthetic air of which $250 \mathrm{ML}$ was pumped onto the first sorbent tube. Then the sampling bag was emptied and subjects held their breath for 5 seconds and then exhaled in the Teflon bag until the bag was filled with approximately $2.5 \mathrm{~L}$. Subsequently the exhaled breath from this Teflon bag was transferred to five sorbent tubes using a breath gas sampler (BGS). The BGS consists of a pump (KNF NMS020B 6VDC Micro Membranegas pump), a mass flow controller (Horiba STEC Z500), battery and charger (Panasonic LC-RA1212PG and IDEAL POWER PC170-2), all combined in a metal casing with an operating display (Brooks Instrument 0254). ${ }^{8}$ Sorbent tubes were made out of stainless steel (6 mm O.D. $\times 7$ in., Supelco, Zwijndrecht, The Netherlands) filled with a combination of Tenax GR (90 mg per tube, Varian Chrompack, Middelburg, The Netherlands) and Carbograph 5TD (300 mg per tube, Markes International Ltd, Llantrisant, UK). A volume of $250 \mathrm{~mL}$ was absorbed on the sorbent tube with a flow of 100-200 $\mathrm{mL} \mathrm{min}^{-1}$ using the BGS.

A $3 \mathrm{~L}$ sampling bag was filled with synthetic air, of which $250 \mathrm{~mL}$ was pumped onto a first sorbent tube. Then, the sampling bag was emptied, and filled with exhaled breath of a healthy subject. From this sampling bag, 5 additional sorbent tubes were loaded ( $250 \mathrm{~mL}$ each), with intermittent addition of predetermined amounts of $n$-octane to achieve spiked breath samples with concentrations of respectively 1, 2, 4 and $8 \mathrm{ppb}$, since the octane level in healthy subjects is very low ${ }^{9}$ (Fig. 1). A sample solely containing exhaled breath served as a baseline. These samples were used to test the accuracy of octane detection.

Breath sampling in invasively ventilated ICU patients. Exhaled breath sampling was performed within 48 hours after start of invasive ventilation. Breath was collected through a disposable side-stream connector, using a polytetrafluoroethylene (PTFE) tube, distal of the heat-moist-exchanger (HME) filter (Fig. 2). Sampling was performed with a fixed flow of $200 \mathrm{~mL}$ $\min ^{-1}$ for 6 minutes using the BGS. Volatile organic compounds (VOCs) were collected on the sorbent tube. Reproducibility of this sampling method has been tested previously and showed a median intra-class correlation of $0.95 .^{10}$ Four sorbent tubes were filled per time point, to create duplicate measurements, two tubes for analysis with the laboratory prototype or POC breath test and two for GC-MS analysis (Table 1). All samples were stored in the fridge. Sorbent tubes analysed during the bench study were transported to the laboratory of Philips (Philips Research, High Tech Campus,

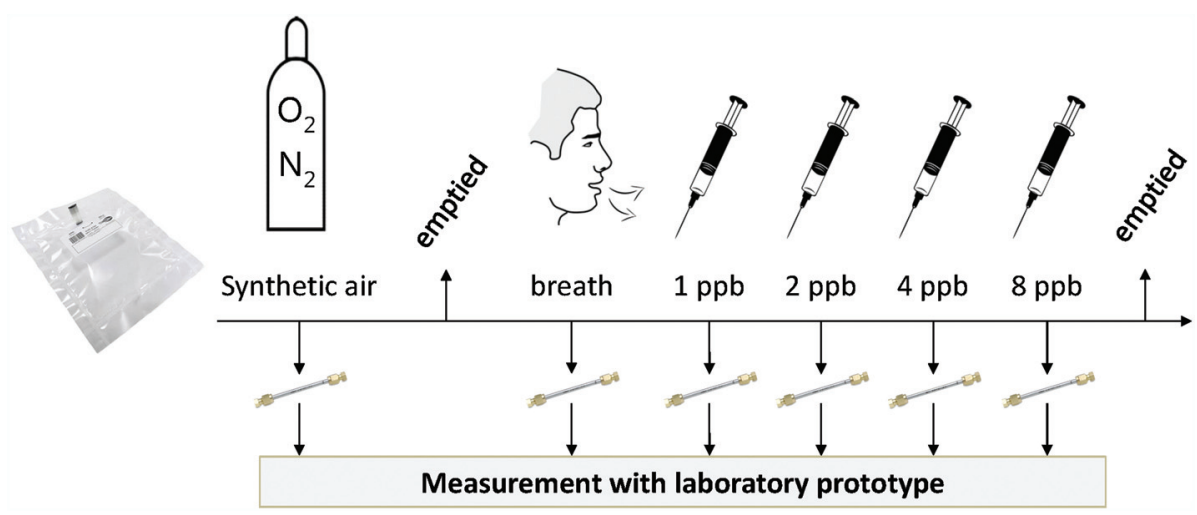

Fig. 1 Sampling method in healthy subjects as measured with the laboratory prototype. A $3 \mathrm{~L}$ sampling bag is filled with synthetic air, of which $250 \mathrm{~mL}$ is pumped onto a first sorbent tube. Then, the sampling bag is emptied, and filled with exhaled breath of a healthy subject. From this sampling bag, 5 additional sorbent tubes were loaded $(250 \mathrm{~mL}$ each), with intermittent addition of predetermined amounts of $n$-octane (from certified permeation tube source) to achieve expected added $n$-octane concentrations of $1,2,4$ and 8 ppb. Afterwards, the remaining volume in the sampling bag was measured to exactly determine the added $n$-octane concentrations. $\mathrm{Ppb}=$ Parts per billion. 


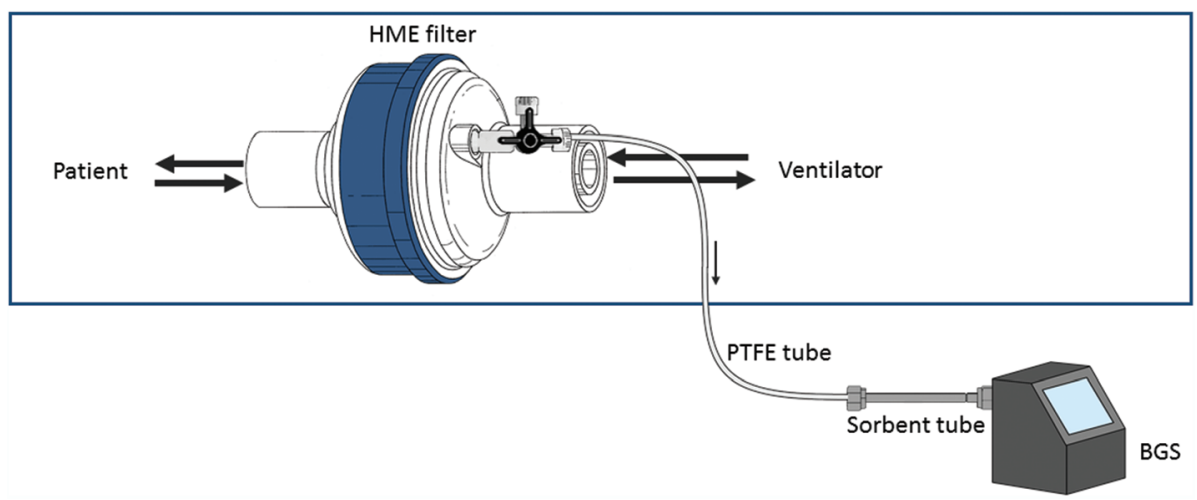

Fig. 2 Breath sampling method in invasively ventilated patients, using the BGS through a side stream connection, distal from the HME filter. VOCs are collected on the sorbent tube. HME = Heat-moist-exchanger; PTFE = polytetrafluoroethylene; BGS = breath gas sampler.

Eindhoven, Netherlands). In the bedside study all analyses were performed in the ICU. All analyses were performed as soon as possible, but within a maximum of two weeks to ensure a stable sample. ${ }^{11,12}$

\section{Exhaled breath analysis}

Laboratory prototype. The laboratory prototype consists of three main parts: a pre-concentrator, a GC column (length $30 \mathrm{~m}$, internal diameter $0.28 \mathrm{~mm}$, film thickness $1 \mu \mathrm{m}, 100 \%$ dimethyl-polysiloxane, Restek United States) and a photoionization detector (PID) (Fig. 3). The PID is custom-made, based on a $10.6 \mathrm{eV}$ UV lamp. A thermal desorber was used to transfer the sample from sorbent tube to the pre-concentrator. Synthetic air, a mixture of oxygen and nitrogen, was used as carrier gas. This removes the need for helium and allows for the possibility to use filtered room air as carrier gas without the need for a gas-cylinder in the near future. Additionally, the use of the PID does not require a vacuum nor a hydrogen supply to operate. The thermal desorber was heated until $180^{\circ} \mathrm{C}$. The pre-concentrator was heated up to $225^{\circ} \mathrm{C}$ to transfer the VOCs onto the GC column for 50 minutes, after which the VOCs were detected with the PID.
The GC column was used with the following temperature program:

- $40{ }^{\circ} \mathrm{C}$ - hold $30 \mathrm{~min}$

- $\operatorname{ramp} 3^{\circ} \mathrm{C} \mathrm{min}^{-1}$ for $10 \mathrm{~min}$ to $70^{\circ} \mathrm{C}$

- hold $6.6 \mathrm{~min}$

- $\operatorname{ramp} 20^{\circ} \mathrm{C} \min ^{-1}$ to $170{ }^{\circ} \mathrm{C}$

- hold 3.4 minutes

- cooling down with ramp $20^{\circ} \mathrm{C} \mathrm{min}^{-1}$ to $40^{\circ} \mathrm{C}$

This program gradually clears the column from all injected VOCs. As the VOCs exit the column, separated according to their volatility and varying interaction with the column coating, they are recorded by the PID that ionizes the VOCs and quantifies the amount of resulting electrical charge. This results in a chromatogram with measured PID intensities over time.

POC breath test. The POC breath test contains the same functional components as the laboratory prototype (Fig. 3). The bounding box external dimensions of the POC breath test are $55 \mathrm{~cm}$ (width) by $53 \mathrm{~cm}$ (depth) and $25 \mathrm{~cm}$ (height).

In the POC breath test the pre-concentrator was heated up to $220^{\circ} \mathrm{C}$, to transfer the VOCs to a sorbent trap and thereafter the VOCs could enter the GC column for 50 minutes, to be subsequently detected with the PID. The temperature program

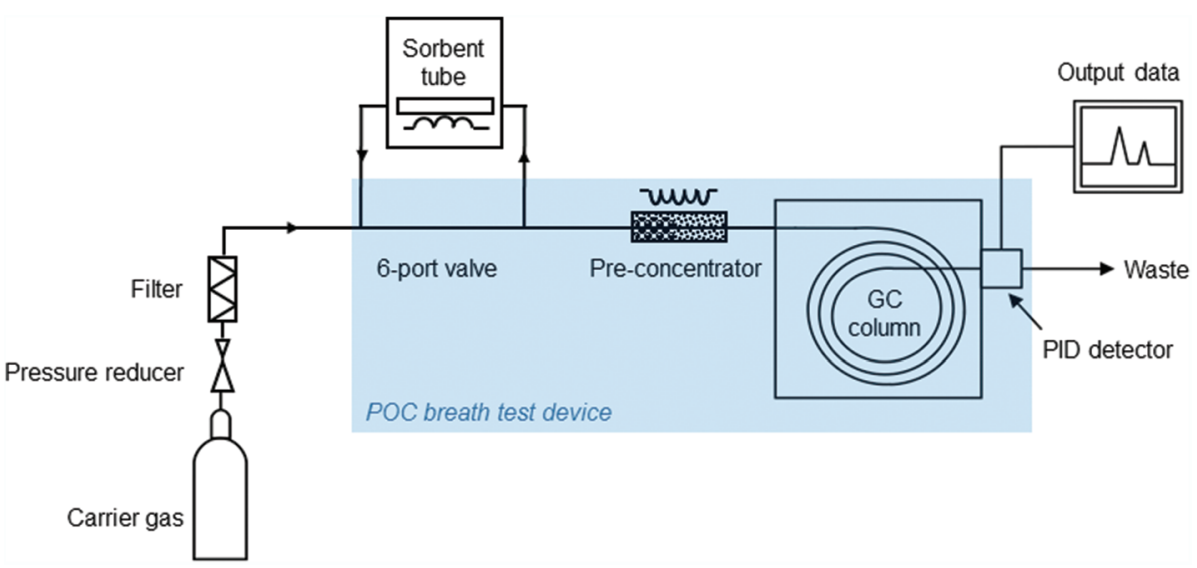

Fig. 3 Schematic overview of parts of the laboratory prototype and POC breath test. POC = Point-of-care; GC = gas chromatography; PID = photoionization detector. 
for the GC column was slightly adapted, the third hold was set to 8.3 minutes instead of 3.4 minutes, to be ensured that all VOCs would have been removed.

Gas chromatography and mass-spectrometry. During the first inclusion period, GC-MS analysis was performed at Philips Research. The sorbent tubes were thermally desorbed at $225^{\circ} \mathrm{C}$ (TDSA, Gerstel, Mülheim an der Ruhr, Germany) into the GC capillary column (6890 N GC, Agilent, SantaClara, CA, USA; column: length $30 \mathrm{~m} \times$ internal diameter $0.25 \mathrm{~mm}$, film thickness $1 \mu \mathrm{m}, \quad 100 \%$ dimethyl-polysiloxane, Varian Chrompack, Middelburg, the Netherlands) and analyser by time-of-flight mass-spectrometry (Pegasus 4D system, LECO, St Joseph, Mi, USA). The GC-MS settings and use of calibration standard were described previously. ${ }^{8}$

During the second inclusion period, GC-MS analysis was performed on-site, according to a protocol described previously. ${ }^{13}$ In short, sorbent tubes were heated to $280{ }^{\circ} \mathrm{C}$ for $15 \mathrm{~min}$ with a flow of $30 \mathrm{ml} \mathrm{min}{ }^{-1}$. VOCs were captured on a cold trap at $10{ }^{\circ} \mathrm{C}$ and then re-injected by rapidly heating the trap to $300{ }^{\circ} \mathrm{C}$ for 1 minute. Subsequently the molecules were injected splitless through a transfer line at $180{ }^{\circ} \mathrm{C}$ onto an Inertcap $5 \mathrm{MS} / \mathrm{Sil}$ GC column [30 m, ID $0.25 \mathrm{~mm}$, film thickness $1 \mu \mathrm{m}, 1,4$-bis(dimethylsiloxy)phenylene dimethyl polysiloxane. (Restek, Breda, The Netherlands)] with a flow of $1.2 \mathrm{ml} \mathrm{min}^{-1}$. Oven temperature was isothermal at $40{ }^{\circ} \mathrm{C}$ for 5 minutes, increased to $280{ }^{\circ} \mathrm{C}$ at $10{ }^{\circ} \mathrm{C} \mathrm{min}^{-1}$, and kept isothermal at $280{ }^{\circ} \mathrm{C}$ for 5 minutes. Molecules were ionized using electron ionization (70 eV), and the fragment ions were detected using a quadrupole mass spectrometer (GCMS-GP2010, Shimadzu, Den Bosch, The Netherlands) with a scan range of 37-300 Da.

GC-MS analysis, de-noising, peak detection, and alignment were performed using the $\mathrm{R}$ "xcms" package (Scripps Center for Metabolomics, La Jolla, CA) and resulted in an ion fragment peak table as input for statistical analysis.
A

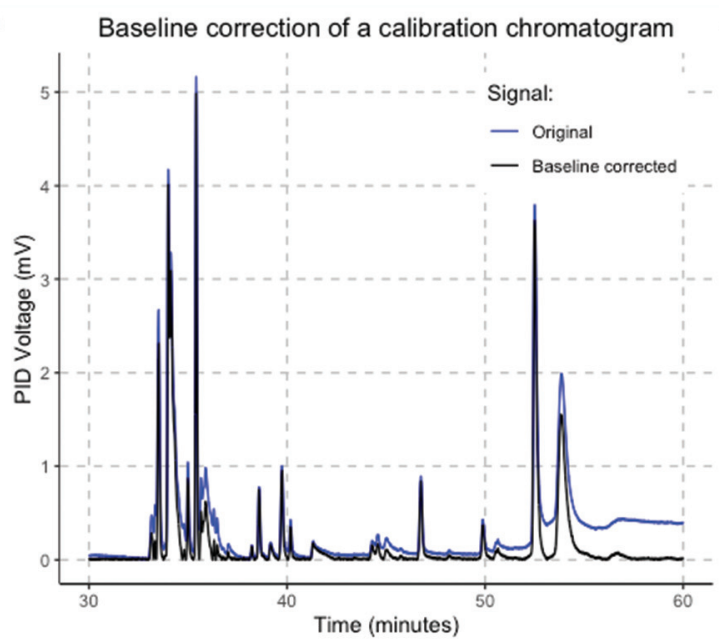

C

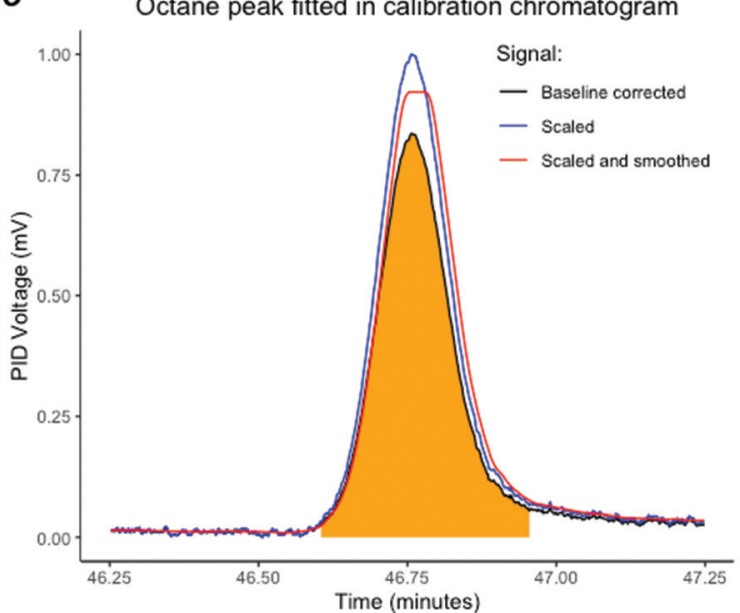

B

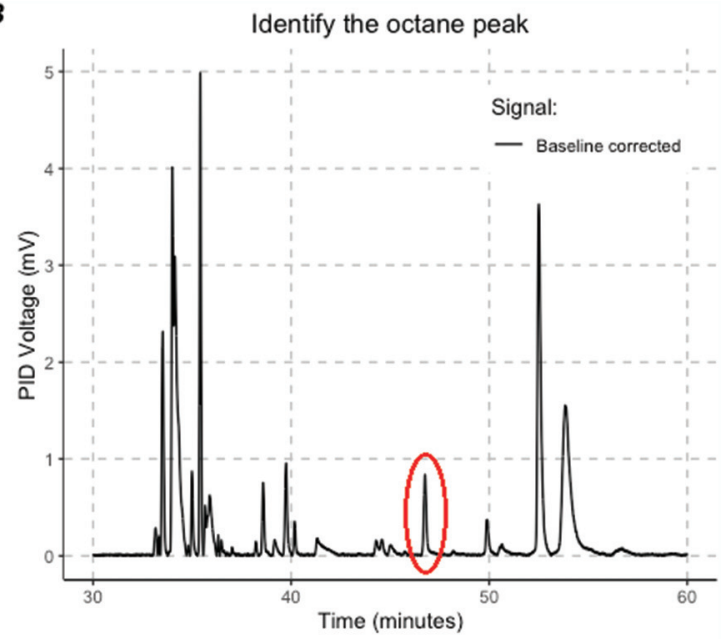

D

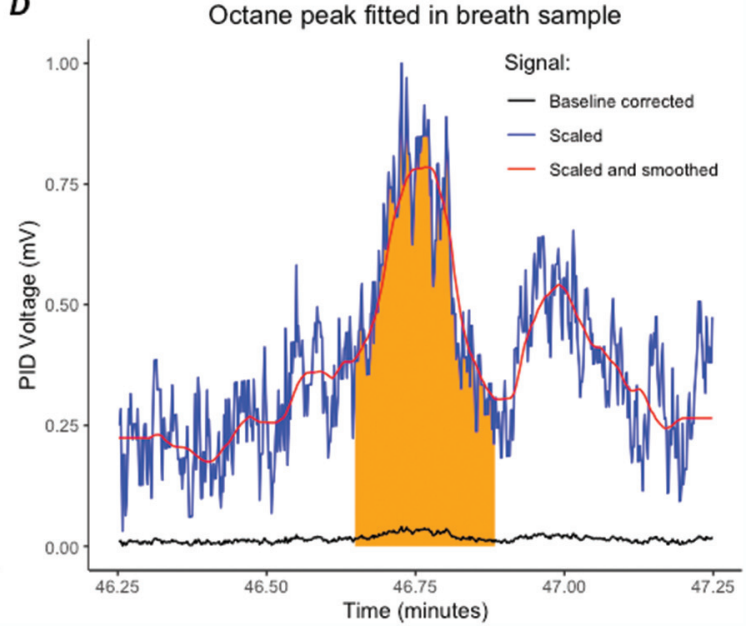

Fig. 4 Steps taken in octane detection. The 30 minutes of holding time is removed from the $x$-axis in figure A and B. The calibration sample shown in figure $A, B$ and $C$ is a sample with $1 \mathrm{ppb}$ octane. The sample in figure $D$ is a patient sample from an invasively ventilated ICU patient. A. Simple baseline correction. B. Identifying the octane peak in a calibration chromatogram. C. Fitting the octane peak in calibration chromatogram. D. Fitting the octane peak in a patient's breath sample. Figure A: Blue: original signal, black: baseline corrected signal. Figure B, C, D: Black: baseline corrected signal. Blue: maximum value in octane window scaled to 1. Red: scaled signal smoothed with a running median and moving average filter for start and end detection. Orange fill: representation of area under the curve (AUC). In figure D this AUC is displayed under the scaled signal, to be able to visualise if start and end detection was correct. 


\section{Octane detection}

For the laboratory prototype analysis was performed manually with MATLAB (MathWorks). For the POC breath test, octane detection was performed using R programming (v3.6.3. http:// www.r-project.org) with the R-studio interface. An algorithm was created to identify and quantify the octane peak in four steps.

First, baseline correction was performed (Fig. 4A). Second, calibration samples that contained a mixture of known molecules with variable volatility were analysed to identify the retention time of octane and to extract the peak characteristics given a prespecified amount of octane (Fig. 4B). The retention time of octane could be determined per sample with a high precision of seconds because there was no co-elution in calibration samples (Fig. 4C). A linear curve was fitted to translate the PID voltage to an octane concentration. By using the expected retention time, octane peaks could be identified and fitted in breath samples (Fig. 4D). The start and end of the peak were determined after using a running median and a moving average filter as noise reduction. The height and area under the peak (AUC) were calculated (Fig. 4D). The concentration of octane in parts per billion (ppbV) was calculated using the standard curve obtained with the calibration samples.

For the GC-MS analysis the octane peak was assessed using the ion-fragment count of mass-over-charge (M/Z) 114 Dalton. A standard curve was fitted on calibration samples and applied to the patient samples.

\section{Statistical analysis}

All statistical analyses were performed in R (v3.6.3. http://www. r-project.org) using the R-studio interface. To assess the precision and validity of the POC breath test, the repeatability and reproducibility (i.e. the agreement with GC-MS outcomes) were assessed. Repeatability was described with mean differences, the coefficient of variation (CV) and the intraclass correlation coefficient (ICC). To visualize the repeatability Bland-Altman plots were used. Reproducibility was described with a Pearson's correlation coefficient.

\section{Results}

\section{Subjects}

For calibration of the laboratory prototype 5 healthy subjects were included. Thereafter, during the clinical evaluation of the laboratory prototype, 40 invasively ventilated ICU patients were enrolled in the bench study (Table 2). To validate and implement the POC breath test on-site, 13 invasively ventilated ICU patients were included (Table 2).

\section{Calibration in healthy subjects}

Octane was added to the breath samples from the 5 healthy subjects to create octane concentrations of 1, 2, 4 and $8 \mathrm{ppb}$, leading to a total of 25 breath samples. To verify these spiked measurements, calibrations samples were used. A positive third order polynomial relation between the expected octane concentration and the AUC of the octane peaks existed for
Table 2 Patient characteristics

\begin{tabular}{lll}
\hline Inclusion period & Period 1 & Period 2 \\
Breath test & Laboratory prototype & POC breath test \\
Number of patients & 40 & 13 \\
Measurements & 40 & 30 \\
Male $N(\%)$ & $30(75.0)$ & $7(54.0)$ \\
Age (years) & $58.0(46.5-68.0)$ & $60.0(60.0-70.0)$ \\
APACHE II & NA & $24(20.0-26.0)$ \\
SOFA score & $10.0(8.0-12.0)$ & $8.0(8.0-11.5)$ \\
$\mathrm{PaO}_{2} / \mathrm{FiO}_{2}(\mathrm{mmHg})$ & $222.5(162.5-293.8)$ & $150.0(117.0-190.0)$ \\
$\mathrm{PEEP}_{\left(\mathrm{cmH}_{2} \mathrm{O}\right)}$ & $6.0(5.0-8.3)$ & $7.0(7.0-8.0)$ \\
$P_{\text {max }}\left(\mathrm{cmH}_{2} \mathrm{O}\right)$ & $21.0(16.5-25.3)$ & $17.0(15.0-19.8)$ \\
Tidal volume $(\mathrm{mL})$ & $537(444-633)$ & $437(402-572)$ \\
& &
\end{tabular}

Variables described as median (IQR) unless else stated.

the calibration samples $\left(R^{2}=0.99\right.$, Fig. 5A). After selecting those samples in the low ppbV range, from $0-2 \mathrm{ppbV}$ which is encountered in clinical practice, a linear relation was found $\left(R^{2}=0.97\right.$, Fig. $\left.5 \mathrm{~B}\right)$. The mean octane concentration found in breath of the healthy subjects was $0.12 \mathrm{ppbV}$ (SD: 0.041).

\section{Validation and implementation of the laboratory prototype}

Breath samples were collected from 40 invasively ventilated patients, leading to 40 duplicate samples for analysis with the laboratory prototype and with the GC-MS (Tables 1 and 2). All octane peaks in the chromatograms of the laboratory prototype could be fitted manually and the AUC could be calculated. The median octane concentration found with the laboratory prototype was $0.15 \mathrm{ppbV}$ (IQR: 0.11-0.20). The repeatability for the measurement of octane with the laboratory prototype showed a difference of $0.02 \mathrm{ppbV}$ (95\% CI -0.091 to 0.13 ) with a coefficient of variation of $17 \%$ (Fig. 6A). The repeated measures showed a correlation coefficient of 0.89 , with an ICC of 0.88 (Fig. 6B). In assessing the reproducibility between the laboratory prototype and GC-MS, 32 measurements were included as GC-MS measurement failed in eight patients. The octane concentrations of the duplicate measurements were averaged for each analysis method. The reproducibility showed a correlation of 0.68 . ICC showed a consistency of 0.72 (95\% CI 0.42-0.86) based on average scores with a two-way model.

\section{Validation and implementation of the POC breath test}

In blank samples no peak for octane could be fitted resulting in an estimated octane concentration of $0 \mathrm{ppb}$ (LoB). The measurements of the breath samples with the POC breath test with a low concentration $(<0.4 \mathrm{ppb})$ of octane showed a standard deviation (SD) of $0.033 \mathrm{ppb}$. Based on the formula LoB +

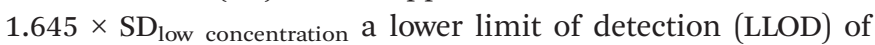
$0.054 \mathrm{ppb}$ was calculated. For the lower limit of quantification (LLOQ) a maximum difference of $0.1 \mathrm{ppb}$ between samples was predetermined as the maximum error. This error limit was met for the whole concentration range, leading to a LLOQ of $0.2 \mathrm{ppb}$.

The (between-run) accuracy of the POC breath test was governed by weekly analysis of calibration samples. This allowed 
$\boldsymbol{A}$

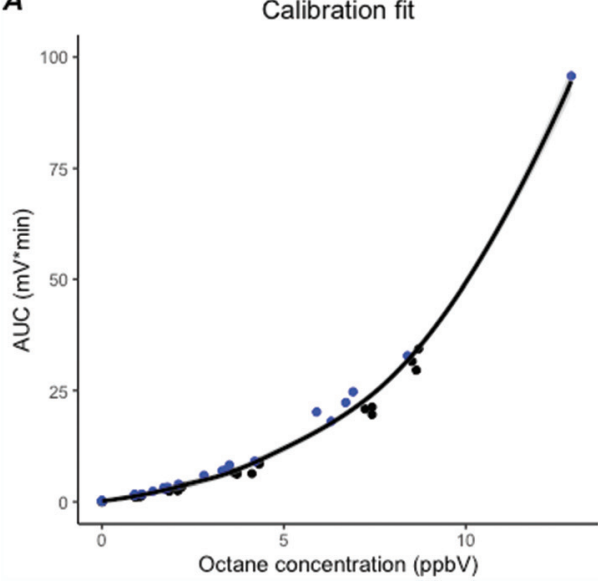

B Calibration fit - Zoom 0-2 ppbV Octane

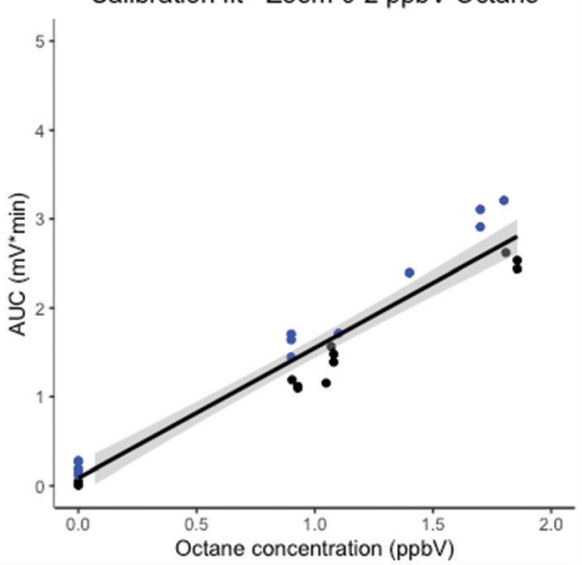

Fig. 5 (A) Calibrations of the laboratory prototype fit almost perfectly on a $3^{\text {rd }}$ order polynomial. Spiked breath samples from healthy subjects are in line with the calibration samples. (B) In the 0-2 ppbV range, relevant for the clinical setting, a linear relation was found. Black: calibration samples. Blue: spiked breath samples. Grey: $95 \%$ confidence interval.
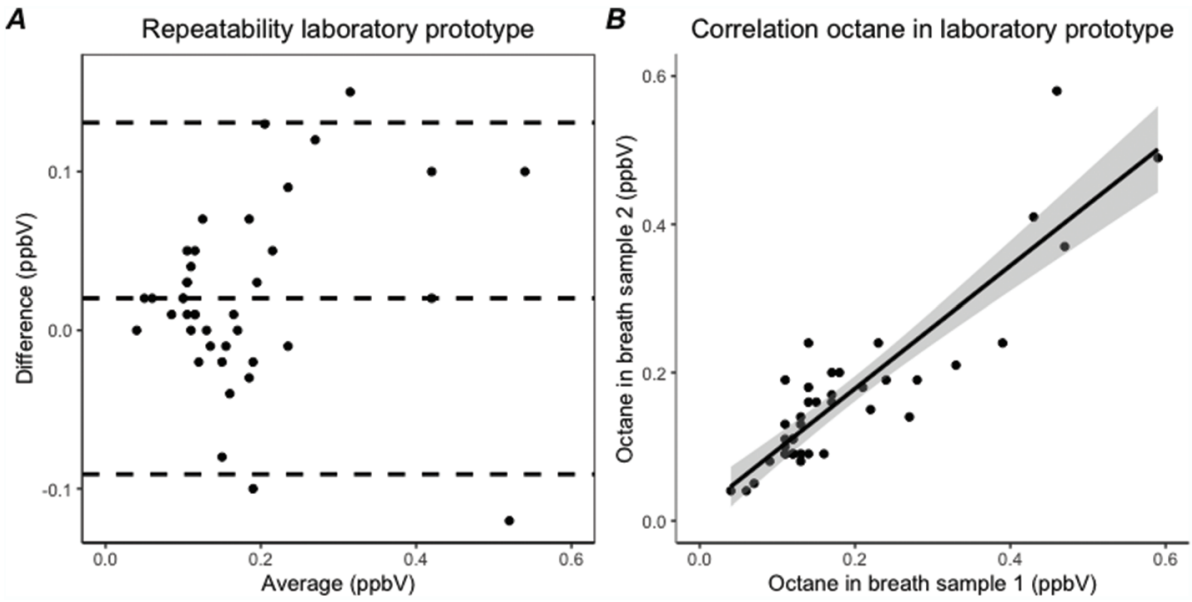

Fig. 6 (A) Bland Altman plot, showing repeatability of the laboratory prototype for 40 duplicate measurements. (B) Correlation plot of 40 duplicate measurements with the laboratory prototype $\left(R^{2}=0.89\right)$. Confidence interval is visualized in grey.

for compensation of the PID lamp output degradation, which was quantified at around $5 \%$ per week of continuous operation. The (between-run) precision of the POC breath test followed from the analysis of multiple calibration samples showing a sample-to-sample variation of about $20 \%$ (2 times SD).

Calibration chromatograms showed no co-elution around the octane peak, enabling accurate detection of the retention time and quantification of the AUC with the POC breath test. This was translatable to patients' samples, allowing proper identification and quantification of octane. Similarly to the laboratory prototype, the AUC obtained with the POC breath test was linearly associated within the range of $0-2 \mathrm{ppbV}$ octane in the calibration samples $\left(R^{2}=1.0\right)$.

During the second inclusion period, duplicate breath samples from 13 invasively ventilated subjects were collected at multiple time points. A total of 30 duplicate measurements were performed, varying from 1 to 4 measurements per patient on different days, resulting in 60 breath samples for analysis.

The created algorithm shown in Fig. 4 was able to correctly classify if an octane peak was present in 57 of the 60 breath samples analysed with the POC breath test (95\%). In the 3 remaining samples the octane peak was missed, due to coelution. In 6 other breath samples, no octane peak was present, so a total of 51 octane peaks were detected by the algorithm. Of these, 49 were correctly characterized (96\%). The two incorrectly characterized peaks and the 3 missed peaks were fitted manually to correctly calculate the AUC, so a total of 54 octane peaks could be included into the analysis. The lower detection limit was imputed when octane was not detected. The median octane concentration was $0.23 \mathrm{ppbV}$ (IQR: 0.21-0.26). The intra-day repeatability was excellent with the POC breath test, with a difference of $0.004 \mathrm{ppbV}$ (95\% CI $-0.06-0.07$ ) and a coefficient of variation of $4.8 \%$ (Fig. 7A). 

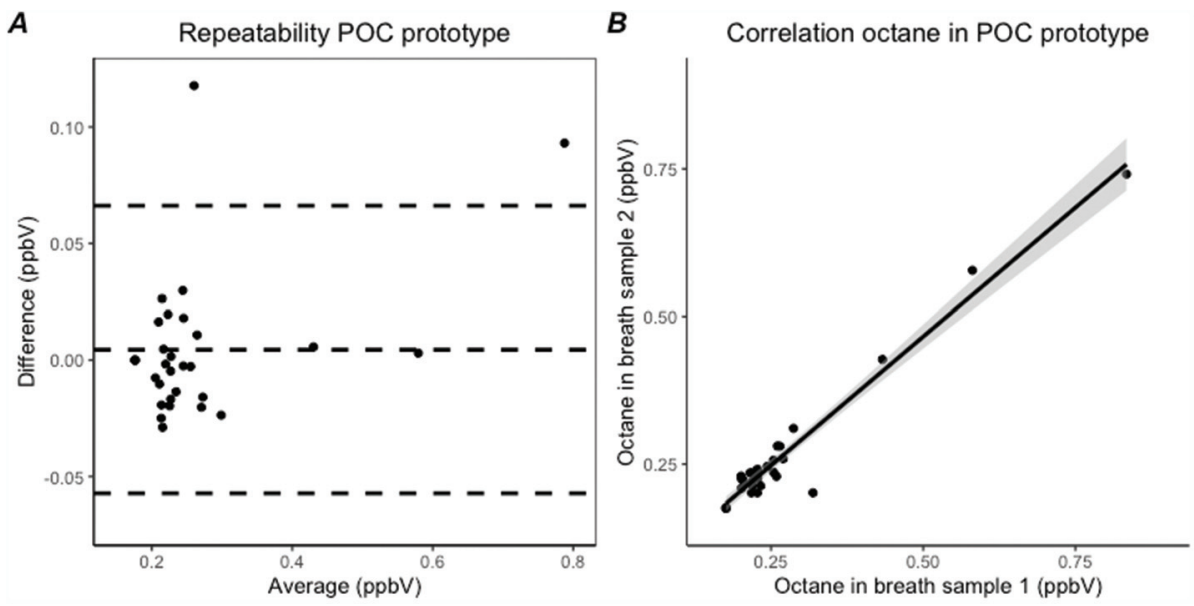

Fig. 7 (A) Bland Altman plot, showing repeatability of the POC breath test for 30 duplicate measurements. (B) Correlation plot of all 30 duplicate measurements with the POC breath test $\left(R^{2}=0.98\right)$. Confidence interval is visualized in grey.

The correlation coefficient was 0.98 and the ICC was 0.97 (95\% CI 0.94-0.99) with a two-way model, type agreement, based on single values (Fig. 7B). Reproducibility with GC-MS showed a correlation coefficient of 0.48 . Consistency between the POC breath test and GC-MS showed an ICC of 0.59 (95\% CI $0.14-0.81$ ) based on average scores with a twoway model.

\section{Discussion}

\section{Summary}

This is the first study to show that a POC breath test can reliably detect octane, with an excellent repeatability, at clinically relevant levels of low ppb in exhaled breath of invasively ventilated ICU patients. Calibration showed a completely separated octane peak, causing a very robust method for the detection of octane in breath samples. Reproducibility with gold standard GC-MS was moderate, probably caused by technical limitations and differences in technical characteristics between the POC breath test and GC-MS.

\section{Comparison to literature}

The developed POC breath test showed to reliably detect exhaled octane concentrations in the sub-ppb range. Other methods frequently have detection limits in the high parts per billion or low parts per million, have a larger footprint or high operating costs, making them unsuitable for POC measurements in the ICU. ${ }^{14-23}$ Compared to an electronic nose, the developed technology has the advantage that a specific VOC, with known association with a particular disease process, can be separated, identified and quantified. This is preferred in the ICU, since pattern recognition based on exhaled breath analysis is very difficult due to the multitude of dynamic changes that are observed in critically ill patients, making it difficult to purse out the pathophysiological meaning of changing patterns. ${ }^{24,25}$
For any chromatographic method, separation of the molecule of interest is of utmost importance. The main challenge with breath samples, particularly from patients undergoing invasive mechanical ventilation, is the variation in observed volatile molecules that may co-elude. Despite our removal of a helium carrier and the move away from massspectrometry, we were able to separate the octane peak from most other molecules frequently encountered in exhaled breath. Therefore, automatic detection of the octane peak in the POC breath test was correct in up to $95 \%$ of observed cases. This is of pivotal importance for clinical application, as rapid results are required in critical care. The total duration from start of sampling to result was less than two hours for this test. Taken together, the developed POC breath test has the advantage that it is small in size, shows rapid results and is easy to use, which makes it more clinically suitable than other robust methods.

To our knowledge, there is only one other breath test that has been described in the literature that also meets these criteria. In this study, the authors used 2-dimension GC coupled with a PID detector to rapidly analyse exhaled breath of invasively ventilated patients. ${ }^{26}$ They also showed detection limits below 1 ppb. The most important differences between that study and ours include the pre-defined molecule of interest in our study with quantitative measurement of the set molecule and a better assessment of repeatability. These studies together show that POC breath analysis is a feasible alternative to laboratory-based technologies.

\section{Strengths and limitations}

This study has important strengths in design and analytical methodology. The POC breath test was validated in the target population for diagnosis of ARDS, namely invasively ventilated ICU patients. By showing reliable measurements in this group, it is directly translatable to clinical studies. Another strength is the use of calibration standards with a wide range of compounds to allow for close monitoring of changes in retention 
time and detector sensitivity. Together, this allows for quantitative assessment of octane in large observational cohort studies. Further, we automated detection of the octane and calculation of the concentration, making the results objective, repeatable and easy to interpret.

In this regard, the POC breath test has different strengths than GC-MS. The strength of GC-MS lies in the accurate identification of unknown VOCs and is therefore used in unbiased studies to identify new biomarkers. Yet, absolute quantification rarely is the goal in such studies. This difference possibly led to a systematic difference and a moderate reproducibility between the GC-MS results and both prototypes of the POC breath test. Part of the difference might be explained by the fact that the detected octane amounts were near the detection limit of the GC-MS, but above the limits of the detection of the POC breath test. A limitation of the POC breath test is the decay in PID intensity. To correct for this decay, regular analysis of the calibration standard is needed.

\section{Interpretation}

Since the POC test can rapidly and reliably detect octane in invasively ventilated patients, it can be studied as a diagnostic tool for the early detection of ARDS. A large observational cohort study that will assess the diagnostic accuracy of octane for ARDS is ongoing.

\section{Clinical implication}

This POC breath test showed reliable quantification of the exhaled concentration of octane. This opens possibilities for targeted exhaled breath analysis to be used as a rapid on-site test in the ICU.

\section{Conclusion}

In conclusion, the POC breath test can reliably detect octane in exhaled breath of invasively ventilated ICU patients. It detects octane accurately, with excellent repeatability at concentrations relevant for the diagnosis of ARDS. Further validation of the diagnostic accuracy for ARDS is needed.

\section{Author contributions}

LDJB and MJS designed the study. LDJB wrote the study protocol. ARMV, NFLH, MRS, DCJJB, RMS, TMEN, IG and LAH advised on the study design and participated in the study protocol. All authors approved the study design. AL, LAH and MRS participated in data collection. Analysis was performed by LAH. LDJB and LAH prepared the initial draft of this manuscript. All authors approved the submitted version of this manuscript.

\section{Conflicts of interest}

There are no conflicts to declare.

\section{References}

1 V. M. Ranieri, G. D. Rubenfeld, B. T. Thompson, et al., Acute respiratory distress syndrome: The Berlin definition, JAMA, 2012, 307(23), 2526-2533, DOI: 10.1001/ jama.2012.5669.

2 G. Bellani, J. G. Laffey, T. Pham, et al., Epidemiology, Patterns of Care, and Mortality for Patients With Acute Respiratory Distress Syndrome in Intensive Care Units in 50 Countries, J. Am. Med. Assoc., 2016, 315(8), 788-800, DOI: 10.1001/jama.2016.0291.

3 G. Bellani, T. Pham and J. G. Laffey, Missed or delayed diagnosis of ARDS: a common and serious problem, Intensive Care Med., 2020, 46(6), 1180-1183, DOI: 10.1007/ s00134-020-06035-0.

4 N. D. Ferguson, E. Fan, L. Camporota, et al., The Berlin definition of ARDS: An expanded rationale, justification, and supplementary material, Intensive Care Med., 2012, 38(10), 1573-1582, DOI: 10.1007/s00134-0122682-1.

5 L. A. Hagens, N. F. L. Heijnen, M. R. Smit, et al., Systematic review of diagnostic methods for Acute Respiratory Distress Syndrome, ERJ Open Res., 2020, 00504-02020, DOI: 10.1183/23120541.00504-2020.

6 L. D. J. Bos, H. Weda, Y. Wang, et al., Exhaled breath metabolomics as a noninvasive diagnostic tool for acute respiratory distress syndrome, Eur. Respir. J., 2014, 44(1), 188197, DOI: 10.1183/09031936.00005614.

7 M. P. van der Schee, T. Paff, P. Brinkman, W. M. C. van Aalderen, E. G. Haarman and P. J. Sterk, Breathomics in lung disease, Chest, 2015, 147(1), 224-231, DOI: 10.1378/ chest.14-0781.

8 P. M. P. van Oort, T. Nijsen, H. Weda, et al., BreathDx molecular analysis of exhaled breath as a diagnostic test for ventilator-associated pneumonia: protocol for a European multicentre observational study, BMC Pulm. Med., 2017, 17(1), 1, DOI: 10.1186/s12890-016-0353-7.

9 P. Mochalski, J. King, M. Klieber, et al., Blood and breath levels of selected volatile organic compounds in healthy volunteers, Analyst, 2013, 138(7), 2134-2145, DOI: 10.1039/ c3an36756h.

10 L. D. J. Bos, Y. Wang, H. Weda, et al., A simple breath sampling method in intubated and mechanically ventilated critically ill patients, Respir. Physiol. Neurobiol., 2014, 191(1), 67-74, DOI: 10.1016/j.resp.2013.11.001.

11 M. P. van der Schee, N. Fens, P. Brinkman, et al., Effect of transportation and storage using sorbent tubes of exhaled breath samples on diagnostic accuracy of electronic nose analysis, J. Breath Res., 2013, 7(1), 16002, DOI: 10.1088/ 1752-7155/7/1/016002.

12 S. W. Harshman, N. Mani, B. A. Geier, et al., Storage stability of exhaled breath on Tenax TA, J. Breath Res., 2016, 10(4), 46008, DOI: 10.1088/1752-7155/10/4/046008.

13 L. D. J. Bos, I. C. Van Walree, A. H. J. Kolk, H.-G. Janssen, P. J. Sterk and M. J. Schultz, Alterations in exhaled breath metabolite-mixtures in two rat models of lipopolysacchar- 
ide-induced lung injury, J. Appl. Physiol., 2013, 115(10), DOI: 10.1152/japplphysiol.00685.2013.

14 M. Phillips, R. N. Cataneo, C. Saunders, P. Hope, P. Schmitt and J. Wai, Volatile biomarkers in the breath of women with breast cancer, J. Breath Res., 2010, 4(2), 26003, DOI: $10.1088 / 1752-7155 / 4 / 2 / 026003$.

15 M. Phillips, V. Basa-Dalay, G. Bothamley, et al., Breath biomarkers of active pulmonary tuberculosis, Tuberculosis, 2010, 90(2), 145-151, DOI: 10.1016/j.tube.2010.01.003.

16 M. Storer, K. Curry, M. Squire, S. Kingham and M. Epton, Breath testing and personal exposure-SIFT-MS detection of breath acetonitrile for exposure monitoring, J. Breath Res., 2015, 9(3), 36006, DOI: 10.1088/1752-7155/9/3/036006.

17 D. Smith and P. Španěl, SIFT-MS and FA-MS methods for ambient gas phase analysis: developments and applications in the UK, Analyst, 2015, 140(8), 2573-2591, DOI: 10.1039/C4AN02049A.

18 P. Spaněl and D. Smith, Progress in SIFT-MS: breath analysis and other applications, Mass Spectrom. Rev., 2011, 30(2), 236-267, DOI: 10.1002/mas.20303.

19 B. Moser, F. Bodrogi, G. Eibl, M. Lechner, J. Rieder and P. Lirk, Mass spectrometric profile of exhaled breath-field study by PTR-MS, Respir. Physiol. Neurobiol., 2005, 145(2-3), 295-300.

20 R. Cumeras, E. Figueras, C. E. Davis, J. I. Baumbach and I. Gràcia, Review on Ion Mobility Spectrometry. Part 1: current instrumentation, Analyst, 2015, 140(5), 1376-1390, DOI: 10.1039/C4AN01100G.
21 J. A. Covington, M. P. van der Schee, A. S. L. Edge, B. Boyle, R. S. Savage and R. P. Arasaradnam, The application of FAIMS gas analysis in medical diagnostics, Analyst, 2015, 140, 6775-6781.

22 M. K. Nakhleh, H. Amal, R. Jeries, et al., Diagnosis and Classification of 17 Diseases from 1404 Subjects via Pattern Analysis of Exhaled Molecules, ACS Nano, 2017, 11(1), 112125, DOI: 10.1021/acsnano.6b04930.

23 N. Queralto, A. N. Berliner, B. Goldsmith, R. Martino, P. Rhodes and S. H. Lim, Detecting cancer by breath volatile organic compound analysis: a review of array-based sensors, J. Breath Res., 2014, 8(2), 27112, DOI: 10.1088/ 1752-7155/8/2/027112.

24 J. H. Leopold, A. Abu-Hanna, C. Colombo, P. J. Sterk, M. J. Schultz and L. D. J. Bos, Factors influencing continuous breath signal in intubated and mechanicallyventilated intensive care unit patients measured by an electronic nose, Sensors, 2016, 16(8), DOI: 10.3390/ s16081337.

25 L. D. J. Bos, M. J. Schultz and P. J. Sterk, Exhaled breath profiling for diagnosing acute respiratory distress syndrome, BMC Pulm. Med., 2014, 14(1), 72, DOI: 10.1186/ 1471-2466-14-72.

26 M. Zhou, R. Sharma, H. Zhu, et al., Rapid breath analysis for acute respiratory distress syndrome diagnostics using a portable two-dimensional gas chromatography device, Anal. Bioanal. Chem., 2019, 411(24), 6435-6447, DOI: 10.1007/s00216-019-02024-5. 